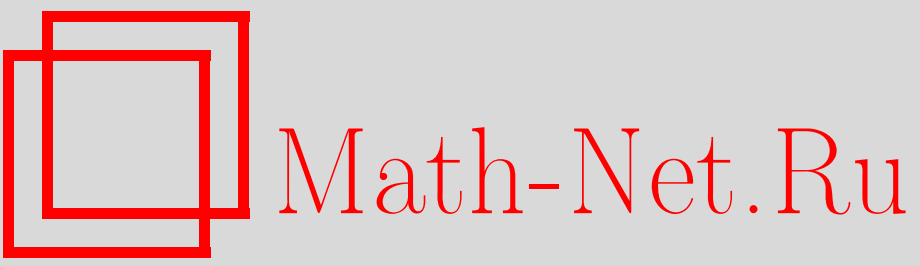

С. Ю. Оревков, М. Г. Зайденберг, О жестких рациональных каспидальных плоских кривых, УМH, 1996, том 51, выпуск 1, 149-150

DOI: https://doi.org/10.4213/rm927

Использование Общероссийского математического портала Math-Net.Ru подразумевает, что вы прочитали и согласны с пользовательским соглашением

http://www.mathnet.ru/rus/agreement

Параметры загрузки:

IP: 54.237 .59 .107

26 апреля 2023 г., 06:25:15 


\title{
О ЖЕСТКИХ РАЦИОНАЛЬНЫХ КАСПИДАЛЬНЫХ ПЛОСКИХ КРИВЫХ
}

\author{
М.Г. ЗАЙДЕНБЕРГ, С. Ю. ОРЕВКОВ
}

Пусть $Y$ - гладкая афффинная алгебраическая поверхность над $\mathbb{C}$. Предположим, что она $\mathbb{Q}$-ациклична, т.е. $H_{i}(Y ; \mathbb{Q})=0, i>0$, и что она $\log$-общего типа, т.е. $\bar{k}(Y)=2$, где $\bar{k}$ логарифмичческая размерность Кодаиры (см. [4], [5], [7]). В [2] поставлен вопрос, является ли такая поверхность жесткой. Жесткость означает, что $h^{1}(\Theta)=0$, где $X$ - минимальное гладкое пополнение поверхности $Y$ дивизором $D$ с простыми нормальными пересечениями (SNC-дивизор), а $\Theta=\Theta_{X}\langle D\rangle$ - логарифмическое касательное расслоение на $X$ вдоль $D$. При $\bar{k}=2$ все известные $\mathbb{Q}$-ацикличные поверхности жестки. Для $\chi=h^{0}-h^{1}+h^{2}$ имеет место $\chi(\Theta)=\left(K_{X}+D\right)^{2}+2($ см. [2, лемма $\left.1.3(5)]\right)$.

1. Формулировка результата. Рассмотрим плоскую неприводимую кривую $D$. Легко видеть, что $Y=\mathbb{P}^{2} \backslash D$ является $\mathbb{Q}$-ацикличной, если и только если $D$ рациональна и каспидальна (мы называем кривую каспидальной, если все ее особенности суть касnь, т.е. аналитически неприводимы). Если $D$ имеет как минимум 3 каспа, то $\bar{k}(Y)=2$ (см. [9]). Жесткость $Y$ эквивалентна проективной жесткости $D$ : любая эквисингулярная вложенная деформация проективно эквивалентна $D[3,(2.1)]$. Жесткость имеет место во всех известных примерах [3]. Настоящая заметка посвящена доказательству следующего факта.

ПрЕДЛОЖенИЕ (1.1) (см. [8]). Проективно жесткая рациональная каспидальная кривая в $\mathbb{P}^{2}$ имеет не более 9 каспов.

2. Логарифмическое неравенство Богомолова-Мияоки-Яо (log-БМЯ). Пусть $D$ - некоторая SNC-кривая на гладкой проективной поверхности $X$, и $Y=X \backslash D$. При $\bar{k}(Y) \geqslant 0$ существует разложение Зарисского $K+D=H+N$, где $H, N$ - $\mathbb{Q}$-дивизоры на $X$ такие, что (i) форма пересечения отрицательно определена на подпространстве $V_{N}$, порожденным неприводимыми компонентами $N$ (в частности, $N^{2} \leqslant 0$ ); (ii) $H C \geqslant 0$ для каждой неприводимой кривой $C \subset X$; (iii) $H$ ортогонален к $V_{N}$ (значит, $\left.(K+D)^{2}=H^{2}+N^{2}\right)$.

Teорема (2.1) [7], [5]. Если $\bar{k}(Y)=2$, mо $H^{2} \leqslant 3 e(Y)$, где e - эйлерова характерисmuкa.

3. Двойственный граф. Пусть $E$ - SNC-кривая на гладкой поверхности с неприводимыми компонентами $E_{1}, \ldots, E_{k}$. Пусть $A_{E}=\left(E_{i} \cdot E_{j}\right)_{i j}$ - ее матрица пересечений. Это то же самое, что матрица иницидентности двойственного графа $\Gamma_{E}$ кривой $E$. Его вершины отвечают неприводимым компонентам $E$, а ребра - их точкам пересечения; вес вершины определен как индекс самопересечения соответствующей компоненты. Положим $d\left(\Gamma_{E}\right)=\operatorname{det}\left(-A_{E}\right)$. Экстремальную линейную ветвь граффа назовем твигом. Крайнюю вершину твига $T$ обозначим через $\operatorname{tip}(T)$. Индуктивность твига $T$ определим как $\operatorname{ind}(T)=d(T-\operatorname{tip}(T)) / d(T)$. Применяя правило Крамера, получаем следующую лемму.

Лемма (3.1). Если $Г$ - взвешенное дерево с $d(\Gamma) \neq 0$, и $B=\left(b_{i j}\right)=A^{-1}$, где $A-$ матрица иницидентности, то $b_{i j}=-d(\Gamma-[i j]) / d(\Gamma)$, где $[i j]-$ минимальный подграф, содержащий $i$-ю и $j$-ю вершинъ.

Комбинируя (3.1) с формулой Якоби для минора обратной матрицы, примененной к $2 \times 2$-минору, отвечающему вершинам $v$ и $v_{0}$, получаем еше одну лемму.

Лемма (3.2). Пусть Г - взвешенное дерево, $T$ - его твиг, иницидентный вериине $v_{0} \in \Gamma-T, u v=\operatorname{tip}(T)$. Положим $d_{T}(\Gamma)=d\left(\Gamma-T-v_{0}\right)$. Тогда $d_{T}(\Gamma)=d(\Gamma-v) d(T)-$ $d(\Gamma) d(T-v)$

СлЕДСтвие (3.3). Eсли $d(\Gamma)=1 u d(T) \neq 0, m o$ ind $(T)=\rceil d_{T}(\Gamma) / d(T)\lceil.($ Здесь $\rceil a\lceil$ означает $\lceil a\rceil-a$, где \lceil\rceil$\rceil:=\min \{n \in \mathbb{Z} \mid n \geqslant a\}$.) 
4. Разложение Пюизо и двойственный граф разрешения. Пусть $C$ - росток плоской неприводимой аналитической кривой в особой точке $p$, и пусть $E=\bigcup E_{i}$ - исключительная кривая минимального разрешения особенности, а $\Gamma$ - двойственный графф кривой $E \cup C$. В подходящих аналитических координатах $C$ имеет вид $x=t^{n}, y=a_{m} t^{m}+a_{m+1} t^{m+1}+\cdots$. Положим $d_{1}=n, m_{i}=\min \left\{j \mid a_{j} \neq 0\right.$ и $\left.j \not \equiv 0\left(\bmod d_{i}\right)\right\}, d_{i+1}=\operatorname{gcd}\left(d_{i}, m_{i}\right)$. Пусть $h$ таково, что $d_{h} \neq 1, d_{h+1}=1$. Положим $r_{1}=m_{1} ; r_{i}=r_{i-1} d_{i-1} / d_{i}+m_{i}-m_{i-1}$ при $i>1$.

ПРЕДЛОЖЕНИЕ (4.1) [1]. (а) Граф Г имеет вид

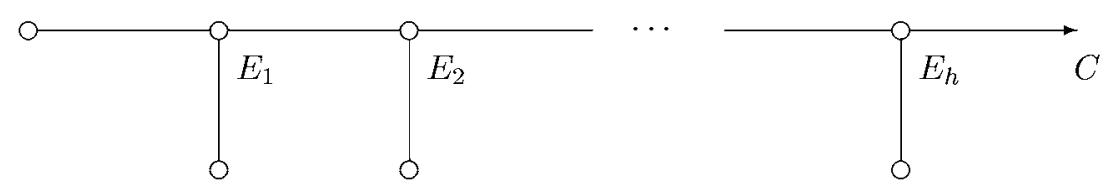

где в виде ребер изображень линейные чепочки вериин.

(б) Пусть $R_{i}, D_{i}$ и $S_{i}$ - компоненты связности графа $\Gamma-E_{i}$, располохсенные соответственно слева, снизу и справа от вериинь $E_{i}$. Тогда $d\left(R_{i}\right)=r_{i} / d_{i+1}, d\left(D_{i}\right)=$ $d_{i} / d_{i+1}, d\left(S_{i}\right)=1$.

Обозначим через $n_{p}$ сумму индуктивностей всех твигов графа $\Gamma$, не содержащих $C$.

СледСТвИЕ (4.2). $\left.n_{p}=\right\rceil d_{1} / r_{1}\left\lceil+\sum_{i=1}^{h}\right\rceil r_{i} / d_{i}\lceil>1 / 2$.

ДокАЗАТЕЛЬСтво. Поскольку $d(\Gamma)=1$, требуемое равенство вытекает из (3.3). Поэтому $\left.n_{p} \geqslant\right\rceil d_{1} / r_{1}\lceil+\rceil r_{1} / d_{1}\lceil$. Очевидно, что если $0<x<1, x \neq 1 / 2$, то $\rceil x\lceil+\rceil 1 / x\lceil>1 / 2$.

5. Пусть $D$ - рациональная каспидальная кривая в $\mathbb{P}^{2}$, и $\sigma: X \rightarrow \mathbb{P}^{2}$ - минимальное разрешение особенностей $D$, т.е. $\widetilde{D}=\sigma^{-1}(D)$ - SNC-дивизор, и $X \backslash \widetilde{D}=Y$. Пусть $K+\widetilde{D}=H+N-$ разложение Зарисского. Обозначим: $S=\operatorname{Sing}(D), s=\# S$.

Лемма (5.1). Если $s \geqslant 3, m o-N^{2}=\sum_{p \in S} n_{p}$.

ДокАЗАТЕЛЬСтво. Поверхность $Y \mathbb{Q}$-ациклична, и при этом в силу [9] мы имеем $\bar{k}(Y)=2$. Поэтому $Y$ не содержит односвязных кривых [10], [6]. Поскольку $s \geqslant 3$, графф $\Gamma_{\widetilde{D}}$ имеет не менее трех точек ветвления. При этих условиях утверждение леммы доказано в [4, (6.20)-(6.24)].

ДокАЗАТЕЛЬство (1.1). Поскольку $\bar{k}(Y)=2$ (см. [9]), то согласно неравенству $\log$-БМЯ $(2.1)$, имеем $H^{2} \leqslant 3$, а значит, по (5.1) и $(4.2),(K+\widetilde{D})^{2}=H^{2}-\sum n_{p}<$ $3-s / 2)$. Пусть $h^{i}=h^{i}\left(\Theta_{X}\langle\widetilde{D}\rangle\right)$. Так как $D$ предполагается жесткой, т.е. $h^{1}=0$, имеем $(K+\widetilde{D})^{2}+2=\chi\left(\Theta_{X}\langle\widetilde{D}\rangle\right)=h^{0}+h^{2} \geqslant 0$. Значит, $s<6-2(K+\widetilde{D})^{2} \leqslant 10$.

\section{СПИСОК ЛИТЕРАТУРЫ}

[1] Eisenbud D., Neumann W.D. // Ann. Math. Stud. V. 110. Princeton: Princeton Univ. Press, 1985. [2] Flenner H., Zaidenberg M. // Contemporary Math. 1964. V. 162. P. 143-208. [3] Flenner H., Zaidenberg M. On a class of rational cuspidal plane curves // Preprint, 1995. P. 1-28. [4] Fujita T. // J. Fac. Sci. Univ. Tokyo (Ser. 1A). 1982. V. 29. P. 503-566. [5] Kobayashi R., Nakamura S., Sakai F. // Proc. Japan Acad. 1989. V. 65(A). P. 238-241. [6] Miyanishi M., Tsunoda S. // J. Math. Kyoto Univ. 1992. V. 32. P. 443-450. [7] Miyaoka Y. // Math. Ann. 1984. V. 268. P. 159-171. [8] Orevkov S. Y., Zaidenberg M. G. // Algebraic Geometry. Proc. Conf., Saintama Univ., March 15-17, 1995 (to appear). [9] Wakabayashi I. // Proc. Japan Acad. 1978. V. 54(A). P. 157-162. [10] Зайденберг М. Г. // Изв. АН СССР. Сер. матем. 1987. Т. 51. С. 534-567; 1991. Т. 55. С. 444-446.

Университет Гренобль I e-mail: zaidenbe@fourier.grenet.fr; НИИ Системных Исследований РАН, Москва e-mail: orevkov@glas.apc.org
Принято редколлегией 01.11 .1995 